\title{
Impact of reviewing lifelogging photos on recalling episodic memories
}

DOI:

10.1145/2968219.2968562

\section{Document Version}

Final published version

Link to publication record in Manchester Research Explorer

\section{Citation for published version (APA):}

Agroudy, P. E., Machulla, T., Rzayev, R., Dingler, T., Funk, M., Schmidt, A., Ward, G., \& Clinch, S. (2016). Impact of reviewing lifelogging photos on recalling episodic memories. In UbiComp '16 Proceedings of the 2016 ACM International Joint Conference on Pervasive and Ubiquitous Computing: Adjunct Publication (pp. 1014-1019). (UbiComp '16 Adjunct). Association for Computing Machinery. https://doi.org/10.1145/2968219.2968562

\section{Published in:}

UbiComp '16 Proceedings of the 2016 ACM International Joint Conference on Pervasive and Ubiquitous Computing: Adjunct Publication

\section{Citing this paper}

Please note that where the full-text provided on Manchester Research Explorer is the Author Accepted Manuscript or Proof version this may differ from the final Published version. If citing, it is advised that you check and use the publisher's definitive version.

\section{General rights}

Copyright and moral rights for the publications made accessible in the Research Explorer are retained by the authors and/or other copyright owners and it is a condition of accessing publications that users recognise and abide by the legal requirements associated with these rights.

\section{Takedown policy}

If you believe that this document breaches copyright please refer to the University of Manchester's Takedown Procedures [http://man.ac.uk/04Y6Bo] or contact uml.scholarlycommunications@manchester.ac.uk providing relevant details, so we can investigate your claim.

\section{OPEN ACCESS}




\section{Impact of Reviewing Lifelogging Photos on Recalling Episodic Memories}

$\begin{array}{ll}\begin{array}{l}\text { Passant El.Agroudy } \\ \text { Tonja Machulla }\end{array} & \begin{array}{l}\text { Geoff Ward } \\ \text { University of Essex } \\ \text { Rufat Rzayev }\end{array} \\ \begin{array}{l}\text { Colchester, UK } \\ \text { Markus Funk }\end{array} \\ \begin{array}{l}\text { Albrecht Schmidt } \\ \text { University of Stuttgart }\end{array} & \begin{array}{l}\text { Sarah Clinch } \\ \text { University of Lancaster }\end{array} \\ \text { Stuttgart, Germany } & \text { Lancaster, UK } \\ \text { firstname.lastname@vis.uni- } & \text { s.clinch@lancaster.ac.uk } \\ \text { stuttgart.de } & \end{array}$

Permission to make digital or hard copies of all or part of this work for personal or classroom use is granted without fee provided that copies are not made or distributed on the first page. Copyrights for components of this work owned by others than on the first page. Copyrights for components ol this work owned by others than ACM to post to post on servers or to redistribute to lists, requires prior specifc permission and fee. Request permissions from Permissions@acm.org.

\begin{abstract}
Photos are a rich and popular form for preserving memories. Thus, they are widely used as cues to augment human memory. Near-continuous capture and sharing of photos have generated a need to summarize and review relevant photos to revive important events. However, there is limited work on exploring how regular reviewing of selected photos influence overall recall of past events. In this paper, we present an experiment to investigate the effect of regular reviewing of egocentric lifelogging photos on the formation and retrieval of autobiographic memories. Our approach protects the privacy of the participants and provides improved validation for their memory performance compared to existing approaches. The results of our experiment are a step towards developing memory shaping algorithms that accentuate or attenuate memories on demand.
\end{abstract}

\section{Author Keywords}

Episodic memory; lifelogging; video summaries; photo reviewing; recall; egocentric photos; retrieval induced forgetting; memory augmentation

\section{ACM Classification Keywords}

H.5.m [Information interfaces and presentation]: Miscellaneous 


\section{FREQUENT TERMS}

"Episodic memory" is the memory of the autobiographical events that occurred in the past and can be stated explicitly [13]. (E.g.: Remembering your emotions during a past trip when you were in high school)

\section{"Reminiscence" is "the} act of recalling past experiences / a mental impression retained or revived"

"Reflection" a is "a thought occurring in consideration or meditation". It means thinking critically about past events.

${ }^{a}$ From www.dictionary.com

"Filtering photos" here refers to removing sensitive or low quality photos then selecting important ones.

"Reviewing photos" here refers to looking at all the photos or a selected subset of them.

\section{Motivation}

Human memory is naturally volatile and prone to errors [11]. As a part of their evolution, humans attempted to preserve their memories using different forms like writing and drawing. Pictures have been a popular medium starting from pharaonic drawings on the walls of temples until cinematic visions of a brain-embedded chip that auto-records the life as a video ${ }^{1}$. Pictures are strong memory cues because they hold condensed and rich details about the context of a situation [8]. They support reminiscence, reflection, search and recall of missing details.

Therefore, numerous commercial platforms have been developed to assist users in capturing and presenting memories through photos. Small and aesthetic wearable cameras like Narrative clip ${ }^{2}$ and iON Snap $\mathrm{Cam}^{3}$ focus on not missing a moment by continuously capturing a first-personview of your life every 30 seconds. The result is massive amounts of photos (ca. 1200 per day). On the other hand, social networks ${ }^{4}$ like Facebook and Instagram empower users to preserve a selected set of highly relevant memories shared among a group of persons.

The downside of the abundant affordable capturing devices, sharing and storage platforms is the crippling large datasets. Filtering and reviewing the produced photos is a time consuming and overwhelming task. Thus, the information retrieval community intervened with solid efforts to automate the selection and presentation of important photos. For example, Gurrin (E.g.: [9]) and Doherty (E.g.: [4]) have done extensive work on developing selection algorithms for key photos and implicit detection of events and activities. On the other hand, Girgensohn (E.g.: [7]) and

\footnotetext{
1 "Final cut" movie (http://www.imdb.com/title/tt0364343/)

2 http://getnarrative.com/

$3 \mathrm{https} / / /$ usa.ioncamera.com/snapcam/

4 www.facebook.com, www.instagram.com
}

Byrne (E.g.: [2]) worked on finding presentation techniques for the generated summaries.

The results of this research were adopted and complemented by the commercial lifelogging platforms to create user experiences that support reminiscence and reflection. For example, Facebook offers an "On this day" application that reviews events from previous years that occurred on the same day. The narrative clip offers an on-demand timelapse video of the photos taken within a day. Also, it automatically clusters the dataset into relevant moments after removing low quality pictures.

Despite the popularity of these applications and the abundance of the photo-reviewing tools, there is limited research using real-world datasets on how the reviewing process shapes our episodic memory (E.g.: Studies using SenseCam like [5] and [12] ). A possible reason is the challenging and unstandardized nature of lifelogging / memory experiments. Privacy concerns about the lifelog photos complicate the recruitment of participants as the photos expose sensitive information. Moreover, there are legal concerns about sharing the lifelogs with researchers because of unconsenting individuals in the photos. Consequently, ground truth for the recalled events is usually missing. Therefore, most well-established frameworks do not require the validation of the narrated events by the participant and operate under the assumption that the provided data is correct (e.g., cognitive interviews [6]). Consequently, these frameworks provide only loose guidelines for extracting memories. Additionally, they require consensus regarding the scoring system as well as cross-rater validation by multiple experts. 


\section{FREQUENT TERMS}

"Retrieval Induced Forgetting (RIF)" refers to the forgetting of some information due to trying to remember other relevant information. While the existence of this phenomenon is well supported by laboratory studies, its reproduction in real-world settings is still subject to on-going research ${ }^{a}$.

a http://recall-fet.eu/2015/07/ recall-at-sarmac-2015/

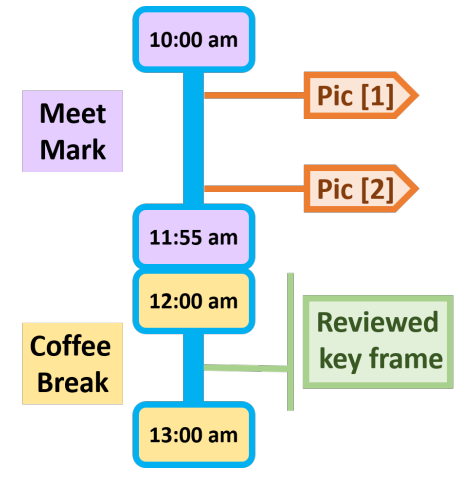

Figure 1: We predict that participants will choose "Pic 2" as a representative for "Meeting Mark" event because it is closer to the reviewed photo.

\section{Vision}

We present an approach to investigate the effect of reviewing lifelogging photos on the formation and retrieval of autobiographic memories. It uses personal lifelogs, protects participants' privacy and obtains subjective validation of the recalled events.

This work is part of the European Union project "RECALL" We aim for creating ubiquitous technologies that augment the human memory. A common approach to memory augmentation is to create an easily searchable and reachable external memory prosthetic that records everything. In contrast, we envision improving the human memory without needing external memory prosthetics.

In the following sections, we will describe the research aims and the design of a planned study. In this study, we predict that the reviewing process will improve the recalling of reviewed events. However, it could also impede the retrieval of unreviewed events as the life of the person is crystallized around the selected photos by the algorithms. The results will provide deeper insights into the role of photoselection systems in shaping our memories and thus perception about life. It also aligns with RECALL's ultimate goal of building context-aware systems that can attenuate/accentuate memories on demand.

\section{Research Aims}

We want to capture potential improvement or deterioration in recalling past events. We will explore the following questions: Does photo reviewing affect the amount of recalled events? The results will indicate the viability of using auto-generated photo reviews as a memory aid to enhance selective recall. We expect that the sheer number of recalled events from the reviewed days is higher than the unreviewed days.

2. Does photo reviewing affect the quality of the recalled events? The focus here is on the amount of recalled details about each event. We will examine if the reviewing promotes recalling richer details about the events.

3. Does photo reviewing affect the recall of close unreviewed events? "Closeness" in this context is the temporal proximity. There are two potential and opposite effects for the reviewing process. The first is better recall of unreviewed events if they are relevant or interconnected with reviewed events. The second one is worse recall of unreviewed events due to the "Retrieval Induced Forgetting" phenomenon [1]. This effect is relevant because it implies that enhancing the memory for some photos (i.e. events) could inhibit remembering other events. This poses an ethical dilemma about the implications of deliberate memory shaping tools as discussed in [3].

4. Are the reviewed photos recognized better than the unreviewed photos? We expect that the reviewed photos are better recognized. Moreover, we predict a tendency to favor the reviewed photos as representatives to the recalled events. We also expect a tendency to choose representative unreviewed photos that are temporally close or spatially similar to the reviewed photos (See Figure 1).

5. Are the recalled events following a pattern? We will explore the data to identify if the free recall is based on a heuristic (E.g.: chronological activity-driven recall). This will enable us to understand more about how the memory works in a natural setup. 


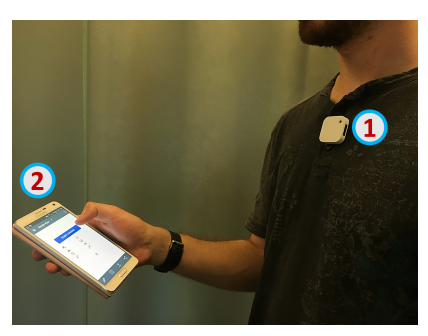

Figure 2: Morning routine for the participants during the experiment. (1) The narrative clip camera is always on, capturing a picture every 30 seconds unless covered. (2) The participant starts the GPS logging explicitly on the smart phone. External GPS is used to overcome the proprietary location format of the camera and to avoid uploading the pictures to narrative cloud service.

\section{Methodology}

We will conduct the study, using a within-study design on 15 participants for 4 weeks each. The participants are mainly university students. Our system is composed of a wearable camera with automatic timelapse mode (E.g. narrative clip), a mobile GPS logger (E.g.: GPS logger mobile app), a dedicated laptop acting as a processing unit and a software for analyzing, selecting and generating a review (summary video). The extraction process of the key pictures is based on the requirements elicited in [8]. The video duration is approximately 2-3 minutes of key pictures. Figure 2 summarizes the morning routine of the participant. The participants can only review a day using our auto-generated video for three days per week. After each review session, they will answer a short questionnaire to subjectively assess the video content.

By the end of each week, the participants are invited to the lab for an individual memory testing session. The session is divided into four phases summarized in Figure 3 :

Phase 1 - Recall events The target is to prompt the participants to recall as many events as they can from the previous week. They are given a fixed interval of time (E.g.: 10 minutes, divided into 1-minute rounds) to recall past events. They note 1-3 words per event as a cue to remember it during the session. This is an adaptation of the technique described in [10].

Phase 2 - Recall details and categorize events The participants are asked to recall specific details about each event (E.g.: time, location, ....) then submit a rating of their certainty about each detail. They also rate the importance of the event and tag it with predefined activities (E.g.: Work, Relax, Family, ...).
Phase 3 - Select a photo per event and validate details Each participant views the photos taken throughout the week on a custom large-screen setup. They pick a photo to represent each event. The system automatically asks them to rate the relevance of the selected photo to the closest two key pictures selected by the algorithm (before and after the selected photo). The participant also reviews his/her answers from phase 2 and validates them.

Phase 4 - Cluster photos to events Each participant reviews the weekly dataset of photos. (S)he groups them into distinct events and tags them with the same predefined tags used in phase 2. Favourite photos are also marked. This phase is optional.

The timestamp of the selected representative photo for each event (in Phase 3) enables us to identify if the photo (i.e. the event) was reviewed or not and infer it's temporal relation to the other events. Thus, we could answer question 1, 3 and 4 . The number of entered details in phase 2 with their validation from phase 3 enables us to answer question 2. Furthermore, the collected tags from phase 2 and phase 4 enables us to answer question 5 .

The proposed design protects the privacy of the participants as the personal data (photos and GPS locations) are stored locally and are accessible by the participant only. We collect only the number of recalled events, photos' names (timestamp format), ratings and miscellaneous computer vision descriptors. Thus, the design offers a trade-off for quantifying abstract concepts, protecting privacy and leveraging meaningful analysis. 


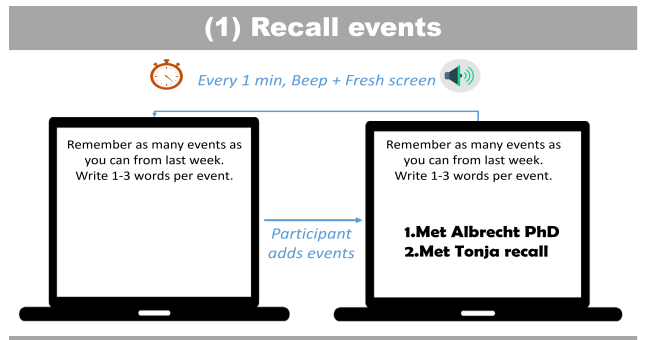

(2) Recall details and Categorize events

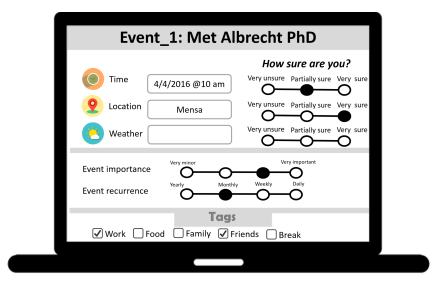

(3) Select a photo per event and validate details

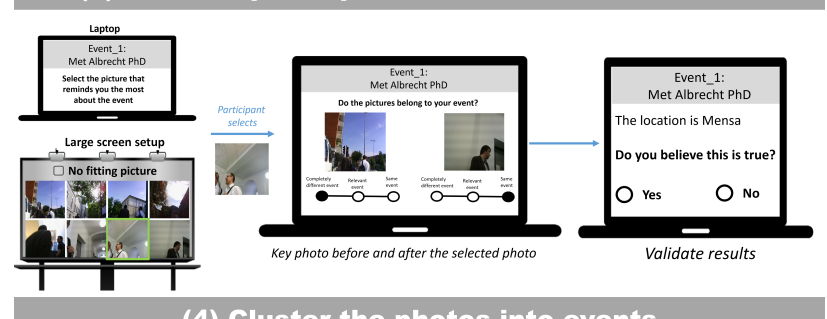

(4) Cluster the photos into events

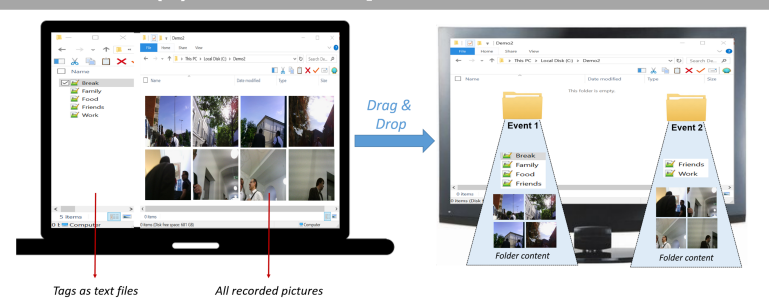

Figure 3: Overview on the memory testing lab session.

\section{Summary}

We propose an experiment to investigate the impact of reviewing selected memories in the form of lifelogging photos on recalling past events. We predict that the reviewing will improve the recall of reviewed events but impede the recall of unreviewed events. The results contribute to understanding the adverse effects of continuous lifelogging and review- ing of memories. Moreover, they support the development of algorithms that control recall on demand.

\section{Acknowledgments}

The authors acknowledge the financial support of the Future and Emerging technologies (FET) programme with the 7th Framework programme for Research of the European Commission, under FET grant number:612933 (RECALL) 


\section{REFERENCES}

1. Anderson, M., Bjork, R., and Bjork, E. Remembering can cause forgetting: retrieval dynamics in long-term memory. Journal of Experimental Psychology:

Learning, Memory, and Cognition 20, 5 (1994), 1063.

2. Byrne, D., Lee, H., Jones, G., and Smeaton, A. Guidelines for the presentation and visualisation of lifelog content. In Proc. iHCl (2008).

3. Dingler, T., and Henze, N. That's the dog from my wedding - algorithms for memory shaping. Workshop Paper. Adjunct Proc. CHI Workshop on Designing Technology for Major Life Events, April 2014.

4. Doherty, A., Byrne, D., Smeaton, A., Jones, G., and Hughes, M. Investigating keyframe selection methods in the novel domain of passively captured visual lifelogs. In Proc. International Conference on Content-based Image and Video Retrieval (New York, NY, USA, 2008), CIVR '08, ACM, pp. 259-268.

5. Finley, J., Brewer, W., and Benjamin, A. The effects of end-of-day picture review and a sensor-based picture capture procedure on autobiographical memory using sensecam. Memory 19, 7 (2011), 796-807.

6. Geiselman, R., Fisher, R., MacKinnon, D., and Holland, $H$. Eyewitness memory enhancement in the police interview: cognitive retrieval mnemonics versus hypnosis. Journal of Applied Psychology 70, 2 (1985), 401.
7. Girgensohn, A. A fast layout algorithm for visual video summaries. In Multimedia and Expo, 2003. ICME '03. Proceedings. 2003 International Conference on (July 2003), vol. 2, pp. II-77-80 vol.2.

8. Le, H., Clinch, S., Sas, C., Dingler, T., Henze, N., and Davies, N. Impact of video summary viewing on episodic memory recall: Design guidelines for video summarizations. In Proc. CHI (New York, NY, USA, 2016), ACM, pp. 4793-4805.

9. Lee, H., Smeaton, A., O'connor, N., Jones, G., Blighe, M., Byrne, D., Doherty, A., and Gurrin, C. Constructing a sensecam visual diary as a media process. Multimedia Syst. 14, 6 (Dec. 2008), 341-349.

10. Moreton, B., and Ward, G. Time scale similarity and long-term memory for autobiographical events Psychonomic Bulletin \& Review 17, 4 (2010), 510-515.

11. Schacter, D. The seven sins of memory, 2001.

12. Sellen, A., Fogg, A., Aitken, M., Hodges, S., Rother, C., and Wood, K. Do life-logging technologies support memory for the past?: an experimental study using sensecam. In Proc. CHI (2007), ACM, pp. 81-90.

13. Tulving, E. What is episodic memory? Current Directions in Psychological Science 2, 3 (1993), 67-70. 